\title{
Mondes du tourisme, une revue ambitieuse au service d'un objet scientifique complexe
}

Le comité de rédaction

\section{OpenEdition}

\section{Journals}

Édition électronique

URL : http://journals.openedition.org/tourisme/312

DOI : $10.4000 /$ tourisme.312

ISSN : 2492-7503

\section{Éditeur}

Éditions touristiques européennes

\section{Édition imprimée}

Date de publication : 1 juin 2010

Pagination : 4-6

ISSN : 2109-5671

\section{Référence électronique}

Le comité de rédaction, " Mondes du tourisme, une revue ambitieuse au service d'un objet scientifique complexe », Mondes du Tourisme [En ligne], 1 | 2010, mis en ligne le 22 septembre 2015, consulté le 22 septembre 2020. URL : http://journals.openedition.org/tourisme/312 ; DOI : https://doi.org/10.4000/ tourisme.312

Ce document a été généré automatiquement le 22 septembre 2020.

\section{cc) $(1) \odot$}

Mondes du tourisme est mis à disposition selon les termes de la licence Creative Commons Attribution - Pas d'Utilisation Commerciale - Pas de Modification 4.0 International. 


\title{
Mondes du tourisme, une revue ambitieuse au service d'un objet scientifique complexe
}

\author{
Le comité de rédaction
}

1 Depuis les années 1970, le phénomène “tourisme" a gagné en importance dans la recherche scientifique. Aujourd'hui, les chercheurs sont dans la situation où la légitimité du tourisme en tant qu'objet de recherche n'est plus à démontrer. Ce changement du contexte scientifique place ces derniers devant de nouveaux enjeux cognitifs, notamment celui d'une conception plus différenciée du phénomène.

Or contexte sociétal a changé aussi. En effet, la transformation de la place de l'objet dans les recherches scientifiques s'accompagne d'un nouvel intérêt des sociétés humaines pour le tourisme, tantôt sujet de toutes les convoitises, tantôt décrié comme producteur de tous les maux. Les chercheurs sont alors confrontés aux injonctions sociales, politiques et économiques qui font rapidement passer la recherche scientifique sur le terrain normatif.

3 Dans le même temps, l'organisation du champ scientifique lui-même s'est modifiée, avec l'émergence de modes de connaissances qui sont partiellement complémentaires, partiellement antagonistes. Dans le contexte actuel d'un dépassement disciplinaire et de la création de nombreux domaines de recherche autour d'objets thématiques (computer science, cultural studies, études rurales, etc.), se pose la question du positionnement $\mathrm{du}$ tourisme dans les sciences sociales. L'émergence d'études touristiques" (tourism studies) ou d'une "science du tourisme" (Tourismus-wissenschaft) fait notamment l'objet de débats. Parallèlement, le tourisme est abordé par plusieurs disciplines issues des sciences sociales, avec l'émergence de spécialités - sociologie du tourisme, anthropologie du tourisme, géographie du tourisme... - qui produisent de nouvelles connaissances. Cette multiplicité de perspectives et de conceptions rend légitime la création d'une revue de recherche scientifique en sciences sociales autour des questions du tourisme. Mondes du tourisme entend ainsi relever le défi du 
croisement des regards disciplinaires, tant en termes de questionnements, de concepts théoriques que de méthodes et de productions de matériaux empiriques.

4 Nous affirmons que le tourisme peut être construit comme objet de connaissance scientifique isolable dans le flux des événements et processus des sociétés humaines. Il est constitué d'un grand nombre d'éléments en interaction tels que les pratiques, les lieux géographiques, les acteurs, les marchés, les régulations, les images, les représentations, les discours, l'imaginaire, etc. Il comporte des dimensions individuelles, sociales, temporelles, spatiales et symboliques; c'est un phénomène complexe. Cette complexité est d'un mode spécifique par l'extrême hétérogénéité des éléments en présence. Ainsi, si le tourisme peut se construire en tant qu'objet spécifique, il peut également s'étudier par ses manifestations dans de nombreuses transformations sociales, culturelles et économiques, dans des imaginaires et projets politiques.

5 Cette posture a deux conséquences. Tout d'abord, le tourisme peut être abordé en tant que tel. Ensuite, il est possible de prendre le tourisme comme une entrée ou un moyen pour analyser toutes sortes de problèmes sociaux, allant de la sociabilité à la production et à la répartition des richesses économiques, en passant par le rapport à l'altérité et à l'identité. Il peut être appréhendé comme "objet social total" qui rend apparents les éléments constitutifs des sociétés humaines.

Cette construction se heurte à des obstacles épistémologiques. La vision du monde et les contextes de production de la connaissance, que ce soit en tant qu'individus ou en tant que collectif, constituent des problèmes intéressants à soulever pour objectiver toute recherche. Cette interrogation du rapport à l'objet - quel travail de terrain ? quel contexte culturel ? - nous semble précieuse et porteuse d'une précision accrue dans la délimitation des conditions de validité des affirmations portées par les chercheurs. Notamment, un "nationalisme méthodologique" peut être relevé, qui conduit souvent à emprunter la perspective des États-nations pour observer le phénomène étudié, et à généraliser des cultures touristiques nationales à toutes les situations observables. Mais les chercheurs se heurtent également à des impositions politiques émanant des institutions. Ainsi, le tourisme a été l'objet d'analyses utilisant sans distance critique des statistiques des producteurs nationaux (offices de statistique) ou internationaux (Organisation mondiale $d u$ tourisme). Mondes $d u$ tourisme souhaite encourager la production de réflexions et de productions empiriques et de données statistiques robustes dépassant les cadres nationaux et européano-centrés.

7 Se pose cependant la question de la définition du tourisme, et ce de plusieurs points de vue. D'abord, la distinction classique entre loisirs et tourisme rencontre de fait une différenciation accrue des pratiques de "re-création" et des rapports à la "recréation". Travailler à trouver les mots qui conviennent à ce nouveau "spectre du loisir" (pour reprendre l'analyse de Norbert Elias) est l'une des ambitions de la revue. Ainsi, des passerelles entre travaux portant sur le tourisme, les loisirs, les pratiques culturelles et sportives sont envisageables.

8 Ensuite, la perspective adoptée lorsque le concept "tourisme" est utilisé en sciences sociales reste hétérogène, donc source de questionnements. La question de savoir si le tourisme est une activité culturelle, un secteur économique, un monde social, un champ ou un système relativement autonome ou simplement une intentionnalité ou un regard reste posée. Le débat autour de l'objet et des différentes conceptions du tourisme nous semble d'une grande importance, et nous souhaitons l'encourager. 
9 Ce problème de définition s'insère dans le contexte de la transformation du tourisme. Depuis l'émergence $d u$ tourisme, concomitamment à la révolution industrielle, la constitution des "mondes du tourisme" est soumise à des transformations économiques, sociales, environnementales et politiques qui font évoluer son organisation et ses pratiques, qu'il s'agisse de changements dans le domaine des résidentialités, des mobilités, des consommations, des technologies, des financements, de la gouvernance, des normes sociales... Ces transformations ont pour conséquence un agencement du tourisme qui diffère fondamentalement de celui de ses débuts. Aujourd'hui, le tourisme n'est plus l'antimonde du travail, il s'insère dans tous les interstices possibles. Il devient urgent alors d'analyser ces changements par rapport aux différents processus sociaux tels que l'avènement du numérique, la remise en cause du niveau d'échelle nationale et l'affirmation d'une société-monde, la métropolisation et la financiarisation de l'économie, l'argument écologique ou encore la mobilité spatiale accrue, l'individualisation, les modes de consommation, les systèmes productifs, ainsi que les accessibilités nouvelles favorisant les déplacements des touristes. Les connaissances produites concernant le tourisme ont aussi pour souci d'éclairer d'autres éléments du monde sociétal. Ainsi, il ne s'agit pas seulement de cantonner l'analyse au phénomène en soi, mais aussi de l'appréhender en tant que révélateur des logiques sociétales.

Mondes du tourisme conçoit plusieurs enjeux pour la recherche scientifique sur le tourisme. Encourager l'invention de nouveaux cadres théoriques ou concepts afin de mieux cerner les processus à l'œuvre est au cœur du projet scientifique et éditorial de la revue. Nous affirmons ainsi la nécessité de continuer à réfléchir sur les fondements du tourisme - entre le désir d'ailleurs, de découverte, de repos, de jeu - qui constituent un problème cognitif à résoudre. Nous faisons appel à l'audace des chercheurs afin qu'ils proposent des questionnements, solutions, concepts hors des sentiers battus. La tâche du chercheur est non seulement la recherche de la plus grande exactitude possible des données empiriques, mais aussi celle de l'inventivité de concepts. La créativité et la fantaisie d'imaginer des mondes non encore advenus sont au centre du projet de Mondes du tourisme. Penser l'impensé et l'impensable, telle est la tâche que nous assignons à la communauté des scientifiques.

11 Il s'agit aussi d'éclairer d'un jour nouveau les phénomènes empiriques connus. Ce renouvellement des cadres d'analyse doit, par exemple, permettre d'appréhender la tension réciproque entre logique individuelle et logique sociale dans les pratiques touristiques, les contradictions entre discours et pratiques de la part des différents acteurs, l'assemblage plus ou moins complexe des pratiques touristiques et des cultures touristiques, la construction d'espaces proprement “touristiques", l'utilisation du tourisme dans les politiques territoriales ou sectorielles, la production de richesses économiques, les rivalités autour de l'usage de ressources, les qualités changeantes de lieux géographiques, l'émergence d'un écoumène touristique mondial, la communication touristique dans toute sa variété, ainsi que les crises globales et locales engendrées ou rencontrées par le tourisme.

12 Mondes du tourisme publie des travaux qui peuvent concerner l'ensemble de la chaîne de production de la connaissance scientifique. Il peut s'agir d'analyses épistémologiques où sont interrogés le rapport à l'objet, la construction du champ de recherche ou le traitement du tourisme par les différentes disciplines. Nous donnons également une place importante aux problèmes méthodologiques qui proposent des 
protocoles de recherche pour l'analyse des phénomènes touristiques, ainsi qu'aux travaux qui se concentrent sur l'évaluation et la mise en œuvre de techniques de constitution du matériau empirique, qu'il s'agisse de production de données d'ordre quantitatif ou qualitatif. Il sera à terme possible de publier ces données - audio, vidéo, image, texte, statistiques - sur le site internet de la revue afin qu'elles soient partagées avec la communauté scientifique.

Les travaux qui se concentrent sur l'analyse ou l'interprétation du matériau empirique sont également les bienvenus car, au-delà d'outils statistiques ou d'analyse de discours ou d'image bien connus, nous souffrons d'un manque de techniques d'analyse sortant des sentiers battus. Nous encourageons enfin des travaux d'ordre théorico-empirique sur les transformations du tourisme et des loisirs, étudiées pour elles-mêmes ou en tant que témoins plus larges d'évolutions du monde contemporain. Nous insistons sur l'indispensable lien entre cadre théorique ou conceptuel et le phénomène empirique analysé, quelle que soit la façon dont est fait ce lien. Les mots qui construisent les choses ont en effet un sens; il importe d'éliminer, autant que faire se peut, l'arbitraire dans le choix des mots dans la chaîne argumentative, explicative et descriptive. Peu importe qu'il s'agisse du bottom-up (comme pour la "grounded theory" où la construction théorique se fait a posteriori) ou du top-down par la pose d'un cadre théorique a priori, ou encore de la modélisation idéal-typique. Nous insistons sur la nécessité de la qualité du matériau empirique, qu'il soit issu de méthodes de collecte quantitatives ou qualitatives. Nous encourageons enfin la publication de descriptions approfondies (thick descriptions) des situations et assemblages touristiques.

14 Mondes du tourisme se conçoit comme le carrefour de travaux scientifiques issus des disciplines en sciences sociales qui s'intéressent aux loisirs et au tourisme (aménagement et urbanisme, anthropologie, économie, géographie, histoire, science de la gestion, science de la communication, science politique, sociologie...). Elle se veut également une passerelle entre travaux francophones et non francophones. Nous constatons, en effet, un hiatus entre les productions francophones, ignorantes de ce qui se passe dans le monde de la recherche anglophone, et les productions anglophones, indifférentes à ce qui se passe dans le monde francophone. Nous cherchons à ouvrir le dialogue entre différentes cultures scientifiques et linguistiques. Le comité scientifique de Mondes du tourisme, qui comporte des personnalités issues de différents pays et institutions, est le garant de tels échanges.

15 Mondes du tourisme fait appel à la créativité des chercheurs pour qu'ils osent produire des travaux qui visent à renouveler les cadres d'analyse et sortent des analyses paradigmatiquement ou politiquement correctes ou à la mode pour produire des innovations scientifiques. 